\title{
Fault-Tolerant Region-Based Control of an Underwater Vehicle with Kinematically Redundant Thrusters
}

\author{
Zool H. Ismail,, ${ }^{1}$ Ahmad 'A. Faudzi, ${ }^{1}$ and Matthew W. Dunnigan² \\ ${ }^{1}$ Centre for Artificial Intelligence \& Robotics, Universiti Teknologi Malaysia, Jalan Semarak, 54100 Kuala Lumpur, Malaysia \\ ${ }^{2}$ School of Engineering and Physical Sciences, Heriot-Watt University, Edinburgh EH14 4AS, UK \\ Correspondence should be addressed to Zool H. Ismail; zool@fke.utm.my
}

Received 20 January 2014; Revised 2 May 2014; Accepted 20 May 2014; Published 11 June 2014

Academic Editor: Mingcong Deng

Copyright (c) 2014 Zool H. Ismail et al. This is an open access article distributed under the Creative Commons Attribution License, which permits unrestricted use, distribution, and reproduction in any medium, provided the original work is properly cited.

\begin{abstract}
This paper presents a new control approach for an underwater vehicle with a kinematically redundant thruster system. This control scheme is derived based on a fault-tolerant decomposition for thruster force allocation and a region control scheme for the tracking objective. Given a redundant thruster system, that is, six or more pairs of thrusters are used, the proposed redundancy resolution and region control scheme determine the number of thruster faults, as well as providing the reference thruster forces in order to keep the underwater vehicle within the desired region. The stability of the presented control law is proven in the sense of a Lyapunov function. Numerical simulations are performed with an omnidirectional underwater vehicle and the results of the proposed scheme illustrate the effectiveness in terms of optimizing the thruster forces.
\end{abstract}

\section{Introduction}

For several decades, extensive efforts have been dedicated to the development of autonomous underwater vehicles (AUVs) for subsea intervention/inspection (primarily the oil and gas industries), scientific exploration, and military purposes. The nature of an AUV, that is, self-contained and capable of performing multipurpose missions, permits it to operate over a wide area without an umbilical cable and human intervention. University and research laboratories became interested in these vehicles and a survey of the research area was given in [1].

Numerous tracking control schemes for an AUV have been reported over the past few years including adaptive control [2,3], robust control [4], and learning control [5-7]. The aforementioned research works focused on motion errors to keep the vehicle on its desired trajectory. It is interesting to note that a small disturbance such as a wave or sea current would pull the vehicle off the actual trajectory and hence high control effort is needed to keep the vehicle on its trajectory as well as maintaining the velocity of the vehicle. Recently, an adaptive region tracking scheme for an AUV was proposed by Li et al. [8] to ensure that the vehicle is kept in a specific region. As opposed to a conventional control method, the presented controller sends command signals for propulsion only when the vehicle is outside the desired region. Therefore, the propellers require less energy to ensure that the vehicle is kept inside the region. Therefore, an arbitrarily small region size implies that a high control effort is required for the AUV.

In the unstructured and hazardous ocean environment, an underwater vehicle is liable to experience faults or failures while executing underwater tasks. Typically, thrusters are known to be one of the most likely sources of faults. In certain cases, the existence of a thruster fault results in the termination of the ongoing mission. The implication of these faults could be very expensive and time consuming. Therefore, research and development to produce a fault-tolerant system for the AUV has gained much attention over the past few years. The study of thruster faults on an underwater vehicle with a kinematically redundant thruster system was reported in [9-13]. This type of AUV has no control surface and as an alternative has more thrusters than the required degrees of freedoms (DOFs) to allow hovering and motion in any direction. Additionally, the thruster redundancy allows 
the hovering AUV to complete the given mission and to return to the support vessel, even though one or more thrusters have malfunctioned. In $[9,10]$, a minimum norm solution with a task space control technique is introduced to solve the problem of optimal thruster force allocation for an overactuated underwater vehicle. The excessive number of thrusters is exploited in order to improve the control performance with the thruster fault. This approach resolves the thruster redundancy in the Cartesian space and allows the AUV to track the task space trajectories with asymptotic reduction of the task space errors. Even though previous works have proposed a range of criterions in the thrust allocation, there is still a significant requirement to maintain high thrust demand for a particular thruster since the vehicle must be kept at various positions on the trajectory. In fact, the presented solution for the control allocation problem and tracking method, that is, computed torque controller $[9,10]$ and PID control [11], do not necessarily reduce the required forces of the individual thrusts and thus possibly generate thrust demands that may exceed an individual thruster's saturation point. In [13], a fuzzy credit assignment cerebellar model articulation controller (FCA-CMAC) neural network with the weighted pseudoinverse approach was recently presented for fault identification and fault-tolerant control of an actuated underwater vehicle. Nevertheless, the proposed method only aims to maintain the propulsion forces and moments at the desired set point with the unanticipated faults.

In this paper, an adaptive region tracking control scheme is proposed for an underwater vehicle with thruster redundancy. Within the proposed control framework, a minimum norm criterion for thruster force distribution is utilized to take account of the thruster faults during the tracking motion. Due to the redundancy, the AUV can still perform the tracking task even though one or more thrusters have malfunctioned. The proposed dynamic region control scheme, where it is formulated in task space, aims to reduce the energy consumed by the redundant thrusters and to simplify the allocation of thruster forces. Unlike the conventional tracking control method, the proposed controller activates and sends commands to the functioning thrusters only when the AUV is outside the desired region and hence it significantly reduces energy consumption. It is impossible to obtain an exact knowledge of the dynamic parameters of an underwater vehicle. Therefore, an adaptive term is introduced in the proposed scheme to overcome the parameter uncertainties. To estimate the unknown parameters of the dynamical system while ensuring the robustness of the system against external disturbances, a combined adaptive sliding mode controller can be utilized [14]. In the robust nonlinear control literature $[15,16]$, this method was proven to overcome the drawbacks of conventional adaptive or sliding mode control although it will lead to the computational burden and increase the complexity of system due to large number of unknown parameters. The rest of the paper is organized as follows. Section 2 describes the kinematics and dynamic properties of an underwater vehicle with kinematically redundant thrusters. Section 3 states the adaptive region control law with faulttolerant decomposition. The stability analysis in terms of

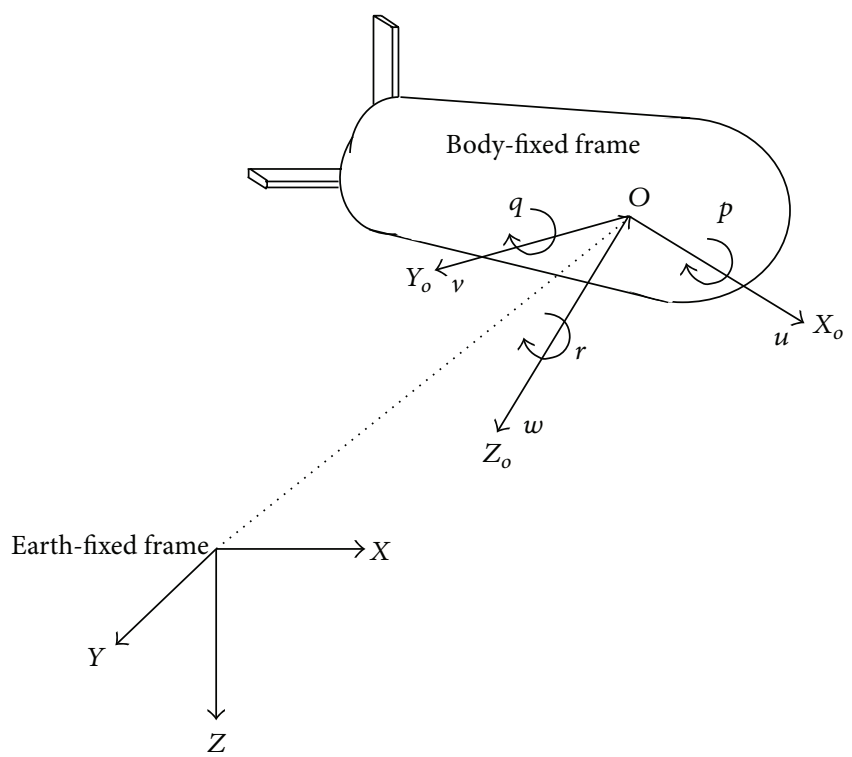

Figure 1: An AUV expressed in body-fixed and earth-fixed reference frames.

the Lyapunov technique is also given in this section. Simulation results are given in Section 4. Finally, Section 5 contains concluding remarks.

\section{Kinematics and Dynamics}

In this section, the structure and properties of an underwater vehicle with kinematically redundant thrusters are briefly discussed. The underwater vehicle state vectors in this paper are conveniently represented according to the Society of Naval Architects and Marine Engineers (SNAME) [17]. The coordinate frames are illustrated in Figure 1.

2.1. Kinematic Model. The relationship between inertial- and body-fixed vehicle velocities can be described using the Jacobian matrix $J\left(\eta_{2}\right)$ in the following form:

$$
\left[\begin{array}{l}
\dot{\eta}_{1} \\
\dot{\eta}_{2}
\end{array}\right]=\left[\begin{array}{cc}
J_{1}\left(\eta_{2}\right) & 0_{3 \times 3} \\
0_{3 \times 3} & J_{2}\left(\eta_{2}\right)
\end{array}\right]\left[\begin{array}{l}
v_{1} \\
v_{2}
\end{array}\right] \Longleftrightarrow \dot{\eta}=J\left(\eta_{2}\right) v
$$

where $\eta_{1}=\left[\begin{array}{lll}x & y & z\end{array}\right]^{T} \in \mathbb{R}^{3}$ and $\eta_{2}=\left[\begin{array}{lll}\phi & \theta & \psi\end{array}\right]^{T} \in$ $\mathbb{R}^{3}$ denote the position and the orientation of the vehicle, respectively, expressed in the inertial-fixed frame. $J_{1}$ and $J_{2}$ are the transformation matrices expressed in terms of the Euler angles. The linear and angular velocity vectors, $v_{1}=$ $\left[\begin{array}{lll}u & v & w\end{array}\right]^{T} \in \mathbb{R}^{3}$ and $v_{2}=\left[\begin{array}{ccc}p & q & r\end{array}\right]^{T} \in \mathbb{R}^{3}$, respectively, are described in terms of the body-fixed frame.

2.2. Dynamic Model. The dynamic equation of motion for an underwater vehicle has been previously investigated in detail [18]. Due to hydrodynamic effects acting on the system such as added mass, drag, lift, and buoyancy forces, the equation becomes highly nonlinear and coupled. Let the velocity state vector with respect to the body-fixed frame be defined by 
$v \in \mathbb{R}^{6}$; the underwater vehicle dynamic equation can be expressed in closed form as

$$
M \dot{v}+C(v) v+D(v) v+g(\eta)=\tau,
$$

where $M$ is the inertia matrix including the added mass term, $C(v)$ represents the matrix of the Coriolis and centripetal forces including the added mass term, $D(v)$ denotes the hydrodynamic damping and lift force, and $g(\eta)$ is the restoring force. For an AUV that has redundant thrusters such that $n>6$, the relationship between the vector of generalized force acting on the vehicle and individual thruster forces can be given as follows:

$$
\tau=E F_{\text {th }}
$$

where $E$ is the thruster configuration matrix and $F_{\text {th }}$ is the desired vector of thruster forces. The matrix $E$ represents the geometry of the AUV and its thruster locations to transform the individual thruster force into generalized forces in the body-fixed frame of the AUV. It is interesting to note that the matrix $E$ is a constant matrix whose elements are the coordinates of the location of each thruster with respect to the center of mass of the AUV. In order to allow the AUV to generate forces for three translational motions and moments for rotational motions, the matrix $E$ is kept at rank 6. Therefore, it is assumed that the AUV in this research work is capable of performing the specific motion in 6-dimensional task space provided that the AUV possesses more degrees of freedom than the minimum number required to execute a given task.

The dynamic equation in (2) preserves the following properties $[2,18]$.

Property 1. The inertia matrix $M$ is symmetric and positive definite such that $M=M^{T}>0$.

Property 2. $C(v)$ is the skew-symmetric matrix such that $C(v)=-C^{T}(v)$.

Property 3. The hydrodynamic damping matrix $D(v)$ is positive definite; that is, $D(v)=D^{T}(v)>0$.

Property 4. The dynamic model as described in (2) is linear in a set of dynamic parameters $\theta_{d} \in \mathbb{R}^{n_{p}}$ and can be written as

$$
M \dot{v}+C(v) v+D(v) v+g(\eta)=Y_{d}(\eta, v, \dot{v}) \theta_{d}
$$

where $Y_{d}(\eta, v, \dot{v}) \in \mathbb{R}^{6 \times n_{p}}$ is a known regression matrix; $n_{p}$ is the total number of physical parameters. It is assumed that if the arguments of $Y_{d}(\cdot)$ are bounded, then $Y_{d}(\cdot)$ is bounded.

\section{Fault-Tolerant Region Tracking Control Scheme}

In this section, a new control law is proposed to achieve the objective of redistribution of the thruster forces between the functioning thrusters in such a way that the AUV is able to track the trajectory of a desired region even if one or more thrusters are faulty. Within the region-based control framework, the desired moving target is specified by a region [19]. A fault-tolerant region tracking control scheme for an AUV is formulated as follows.

First, a dynamic region with specific shape is defined so that the vehicle converges into the region. This can be viewed as a main objective of the underwater vehicle. The objective function for the desired region of the AUV is defined by the following inequality:

$$
f\left(\delta \eta_{B}\right) \leq 0
$$

where $\delta \eta_{B}=B\left(\eta-\eta_{d}\right) \in \mathbb{R}^{6}$ are the continuous first partial derivatives of the dynamic region, $\eta_{d}(t)$ is the time-varying reference point inside the geometric shape, and $B(t)$ is a timevarying and nonsingular scaling factor. It is assumed that $\eta_{d}(t)$ and $B(t)$ are bounded functions of time. To achieve the scaling formation, that is, if the scaling factor increases then the size of a desired region also increases, a nonsingular matrix is defined as follows:

$$
B=\left[\begin{array}{cc}
B_{1} & 0 \\
0 & B_{2}
\end{array}\right]
$$

where $B_{1}$ is the scaling matrix of $\eta_{1}$ and $B_{2}$ is the scaling matrix of $\eta_{2}$. The scaling of the orientation of the AUV is not required in general, so $B_{2}$ can be set as an identity matrix. Thus, the scaling matrix $B_{1}$ is given as

$$
B_{1}=\left[\begin{array}{ccc}
s_{x} & 0 & 0 \\
0 & s_{y} & 0 \\
0 & 0 & s_{z}
\end{array}\right] \text {, }
$$

where $s_{x}(t), s_{y}(t)$, and $s_{z}(t)$ are scaling factors. This function is useful when the AUV needs to adapt the moving region, depending on the situation and environment. The corresponding potential energy function for the desired region described in (5) can be specified as

$$
\begin{aligned}
P\left(\delta \eta_{B}\right) & =\frac{k_{p}}{2}\left[\max \left(0, f\left(\delta \eta_{B}\right)\right)\right]^{2} \\
& \triangleq \begin{cases}0, & f\left(\delta \eta_{B}\right) \leq 0 \\
\frac{k_{p}}{2} f^{2}\left(\delta \eta_{B}\right), & f\left(\delta \eta_{B}\right)>0,\end{cases}
\end{aligned}
$$

where $k_{p}$ is a positive scalar.

Remark 1. The main contribution of this paper is that an approach called the region function approach is formulated to minimize the required forces amongst functioning thrusters rather than the conventional method of tracking control. The region function approach activates the thrusters only when the AUV is outside the desired region and hence it suggests that less energy is needed to perform the given task. To illustrate this, consider an inequality function $f\left(\delta_{x y}\right)=$ $\left(x-x_{d}\right)^{2}+\left(y-y_{d}\right)^{2} \leq r^{2}$, where $s_{x}=s_{y}=1$ and $r$ is a regional bound, expressed in $2 \mathrm{D}$ task space. Then, the associated potential energy can be illustrated in Figure 2(a) where $P\left(\delta \eta_{B}\right) \in C^{2}$ is lower bounded by zero. Next, invoking 


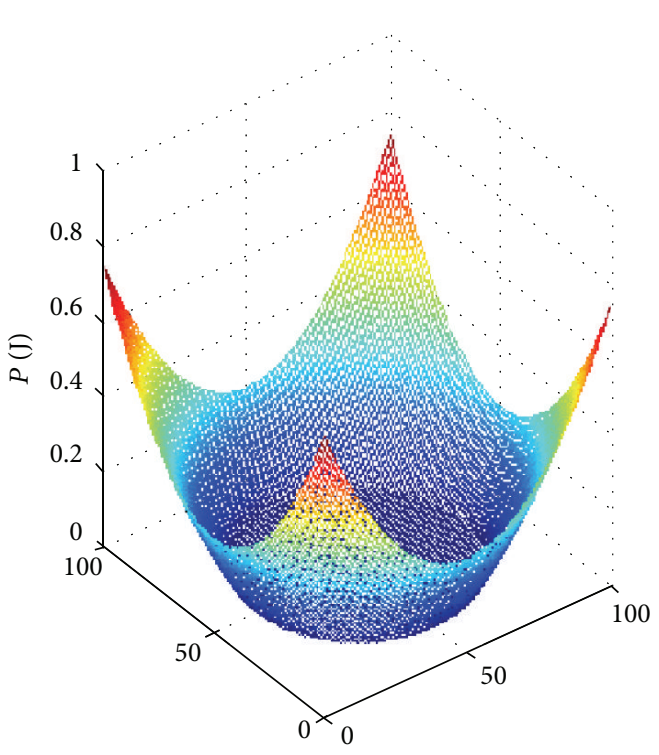

(a)

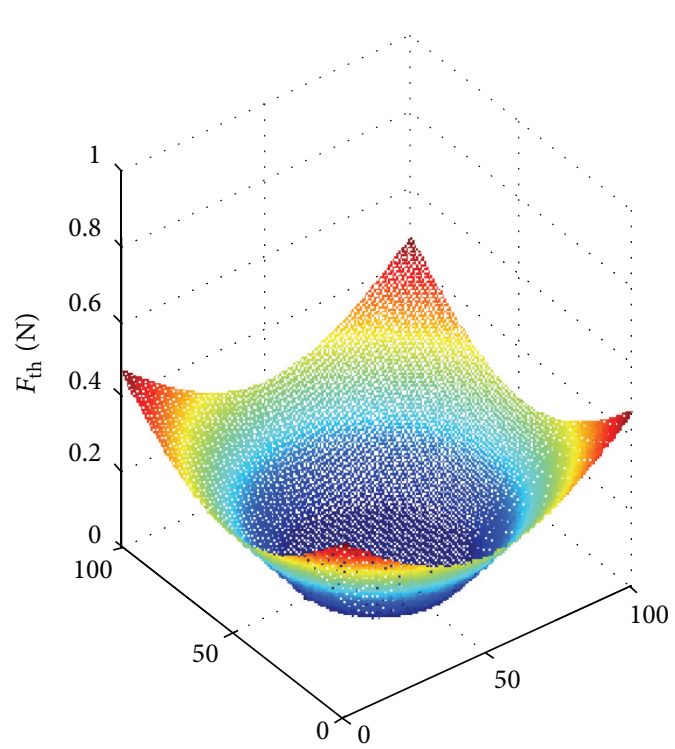

(b)
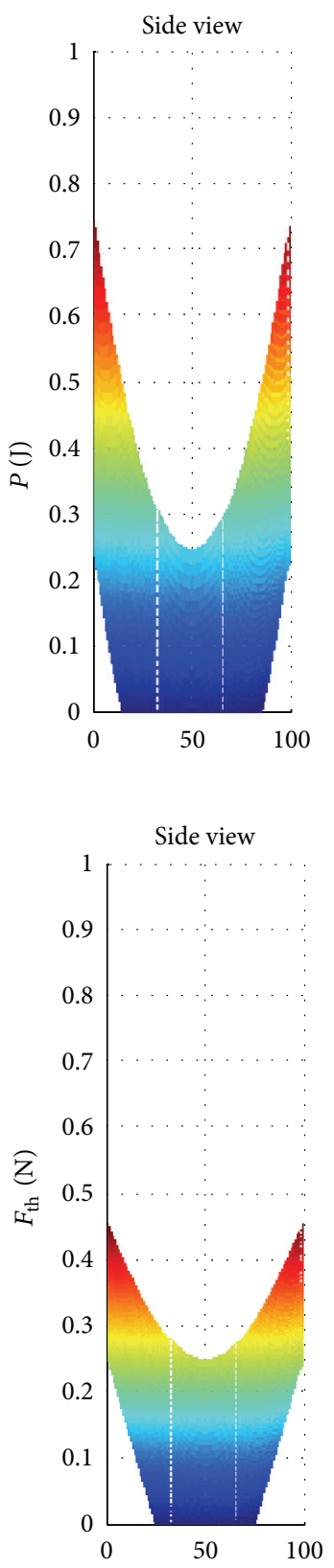

FIGURE 2: An illustration of the required energy in 2D Cartesian space when $r$ is set to a positive constant: (a) potential energy and (b) kinetic energy.

the following linear relationship to obtain the required forces of two onboard thrusters:

$$
F_{\mathrm{th}}=k\left\|f\left(\delta_{x y}\right)\right\|_{2},
$$

where $F_{\text {th }}$ is the net thruster forces, $k$ represents a force constant, $\|\cdot\|_{2}$ is the $\ell_{2}$-norm, and $f\left(\delta_{x y}\right)$ is the displacement in a planar plane. If $k_{p}$ is set to 1 , then the respective forces can be depicted in Figure 2(b). Note that the functioning thrusters consume energy only when the AUV is outside the desired region. In contrast, Figure 3 shows the potential energy and net thruster forces when $r$ is set to be arbitrarily small or the region bound becomes a point. In this case, the onboard thrusters will always be active and, therefore, more energy is needed for each thruster to ensure that the AUV tracks the desired point.

Now, differentiating (8) with respect to $\delta \eta_{B}$ gives

$$
\left(\frac{\partial P\left(\delta \eta_{B}\right)}{\partial \eta_{B}}\right)^{T}=k_{p} \max \left(0, f\left(\delta \eta_{B}\right)\right)\left(\frac{\partial f\left(\delta \eta_{B}\right)}{\partial \eta_{B}}\right)^{T}
$$



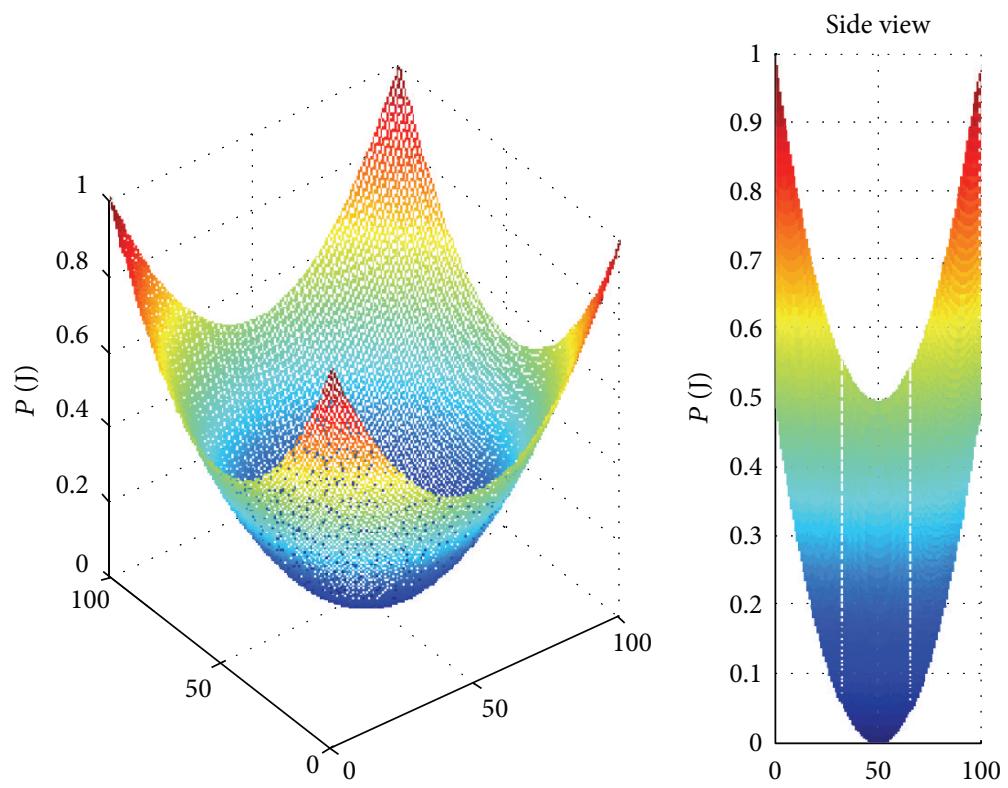

(a)
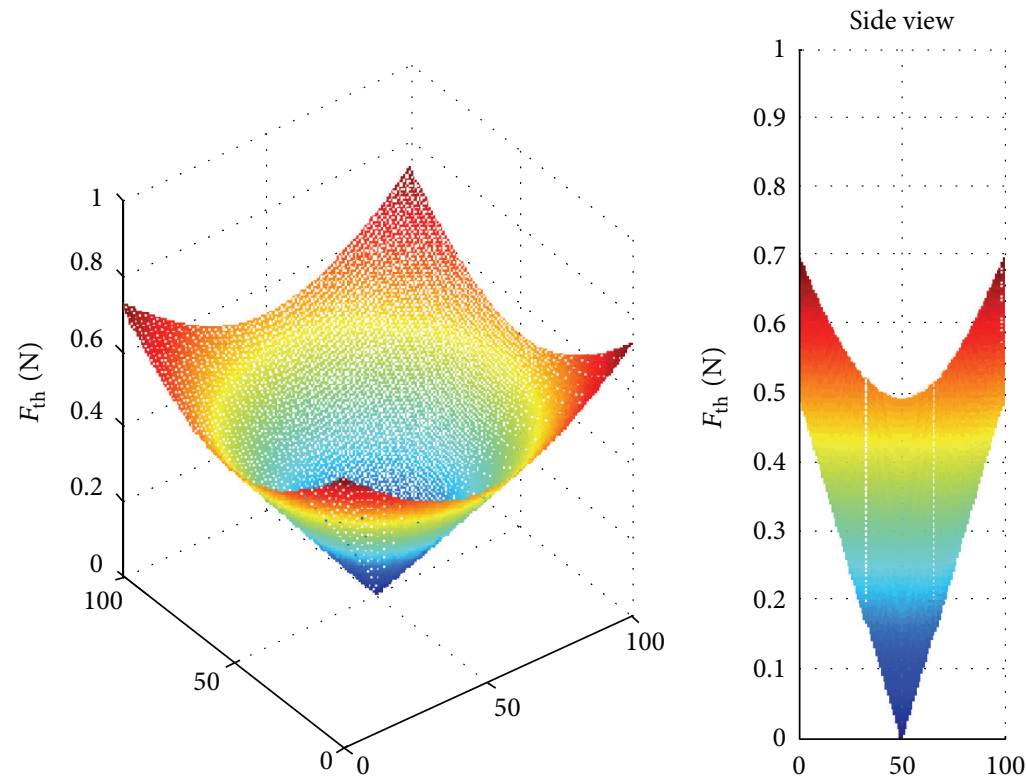

(b)

FIgURE 3: An illustration of the required energy in 2D Cartesian space when $r$ is arbitrarily small: (a) potential energy and (b) kinetic energy.

Now, let (10) be represented as the region error $\widetilde{e}_{B}$ in the following form:

$$
\tilde{e}_{B}=\max \left(0, f\left(\delta \eta_{B}\right)\right)\left(\frac{\partial f\left(\delta \eta_{B}\right)}{\partial \eta_{B}}\right)^{T} .
$$

From the definition of the Cartesian region function, note that $\max \left(0, f\left(\delta \eta_{B}\right)\right)\left(\partial f\left(\delta \eta_{B}\right) / \partial \eta_{B}\right)^{T} \neq 0$ when the AUV is outside the Cartesian region. Once the vehicle reaches the region, this term becomes zero.
Next, a vector $v_{r}$ that is useful is defined as

$$
v_{r}=J^{-1}\left(\dot{\eta}_{d}-B^{-1} \dot{B} \delta \eta_{B}\right)-\alpha J^{-1} B^{-1} \widetilde{e}_{B}
$$

where $\alpha$ is a positive constant. The matrix $J^{-1}$ represents the inverse of the Jacobian matrix, $\dot{B}$ is the time derivative of $B$, and $B^{-1}$ is the inverse of the scaling matrix. From the arguments of trigonometric functions, these matrices are all bounded. Based on the structure of (11) and (12) and the subsequent stability analysis, a filtered tracking error vector for an underwater vehicle is defined as

$$
r(t)=v-J^{-1}\left(\dot{\eta}_{d}-B^{-1} \dot{B} \delta \eta_{B}\right)+\alpha J^{-1} B^{-1} \widetilde{e}_{B} .
$$


In general, the development of the open-loop error system for $r(t)$ can be obtained by premultiplying the inertia matrix with the time derivative of $r(t)$ to yield

$$
M \dot{r}+C(v) r+D(v) r+Y_{d}(\cdot) \theta_{d}=E F_{\text {th }},
$$

where

$$
Y_{d}(\cdot) \theta_{d}=M \dot{v}_{r}+C(v) v_{r}+D(v) v_{r}+g(\eta),
$$

and the derivative of $v_{r}$ in (12) is given as

$$
\begin{aligned}
\dot{v}_{r}= & \dot{J}^{-1}\left(\dot{\eta}_{d}-B^{-1} \dot{B} \delta \eta_{B}\right) \\
& +J^{-1}\left(\ddot{\eta}_{d}-B^{-1} \dot{B} \delta \dot{\eta}_{B}-\left(B^{-1} \ddot{B}+\dot{B}^{-1} \dot{B}\right) \delta \eta_{B}\right) \\
& -\alpha \dot{J}^{-1} B^{-1} \widetilde{e}_{B}-\alpha J^{-1}\left(B^{-1} \dot{\tilde{e}}_{B}+\dot{B}^{-1} \widetilde{e}_{B}\right),
\end{aligned}
$$

where $\dot{\eta}_{d}(t), \ddot{\eta}_{d}(t)$, and $\ddot{B}(t)$ are all assumed to be bounded functions of time. Next, substituting (13) into (2), the following relationship between the derivative of filtered tracking error vector and thruster forces is obtained:

$$
\dot{r}=M^{-1} E F_{\text {th }}-M^{-1}\left[C(v) r+D(v) r+Y_{d}(\cdot) \theta_{d}\right] .
$$

Replacing $\dot{r}$ with $\dot{v}-\dot{v}_{r}$ leads to

$$
\begin{aligned}
\ddot{\eta}-\dot{J} v-J \dot{v}_{r}= & J M^{-1} E F_{\text {th }} \\
& -J M^{-1}\left[C(v) r+D(v) r+Y_{d}(\cdot) \theta_{d}\right],
\end{aligned}
$$

where $\ddot{\eta}=J v+\dot{J} \dot{v}$ and $\dot{v}_{r}$ is defined in (16). Hence, the mapping between task-space acceleration and the thruster forces can be given as

$$
\ddot{\eta}=u F_{\mathrm{th}}-J M^{-1}\left[C(v) r+D(v) r+Y_{d}(\cdot) \theta_{d}\right]+\dot{J} v+J \dot{v}_{r},
$$

where $u=J M^{-1} E$ is a nonsquare thruster control matrix. To find the $\ell_{2}$-norm solution for the desired thruster force $F_{\text {th }}$, the weighted pseudoinverse matrix is utilized as a generalized inverse matrix of $u$ :

$$
\begin{aligned}
F_{\mathrm{th}}= & u^{W \dagger} \ddot{\eta}+u^{W \dagger} J M^{-1}\left[C(v) r+D(v) r+Y_{d}(\cdot) \theta_{d}\right] \\
& -u^{W \dagger}\left(\dot{J} v+J \dot{v}_{r}\right),
\end{aligned}
$$

where $u^{W \dagger}=W^{-1} u^{T}\left(u W^{-1} u^{T}\right)^{-1} ; W$ is a weighted matrix that is used to identify the fault information of each thruster. It is assumed that a faulty thruster(s) has been detected and the detected faulty thruster has completely malfunctioned. Thus, it is also known as the thruster fault matrix [9]. The obtained thruster forces from (20) minimize $(1 / 2) F_{\mathrm{th}}^{T} W F_{\mathrm{th}}$. In the case of no fault in any of the thrusters, the weighted matrix $W$ is an identity matrix. Hence, $u^{W \dagger}=u^{\dagger}$ is a Moore-Penrose pseudoinverse matrix and $(1 / 2) F_{\text {th }}^{T} F_{\text {th }}$ will be minimized. Based on the error system development, the $\ell_{2}$ norm solution for thruster force in (20), and the subsequent stability analysis, the proposed control law for AUV can be formulated as

$$
F_{\text {th }}=u^{W \dagger} J M^{-1}\left[-\alpha J^{T} B \widetilde{e}_{B}-K_{v} r+Y_{d}(\cdot) \widehat{\theta}_{d}\right],
$$

where $K_{v}$ is positive constant matrix.
Remark 2. The $\ell_{\infty}$-norm can be used to optimize the individual thrust force instead of minimizing the $\ell_{2}$-norm. The $\ell_{\infty^{-}}$ norm provides the exact representation of the feasible thrust solution space, while the $\ell_{2}$-norm provides an approximation of the feasible solution space. This suggests that the $\ell_{\infty}$-norm always finds a feasible solution as long as a solution exists within the thrusters' saturation limits [20].

The estimated parameters $\hat{\theta}_{d}$ are updated using the following parameter update law:

$$
\dot{\hat{\theta}}_{d}=-L_{d} Y_{d}^{T}(\cdot) r
$$

where $L_{d}$ is a symmetric positive definite matrix. Substituting (21) into (19) produces a closed-loop dynamic equation for $r(t)$ as follows:

$$
\begin{aligned}
& u^{W \dagger} \ddot{\eta}+u^{W \dagger} J M^{-1}\left[C(v) r+D(v) r+Y_{d}(\cdot) \theta_{d}\right] \\
& -u^{W \dagger}\left(\dot{J} v+J \dot{v}_{r}\right)=u^{W \dagger} J M^{-1}\left[-\alpha J^{T} B \widetilde{e}_{B}-K_{v} r+Y_{d}(\cdot) \widehat{\theta}_{d}\right] .
\end{aligned}
$$

Simplifying (23) leads to

$$
\begin{gathered}
\ddot{\eta}+J M^{-1}\left[C(v) r+D(v) r+Y_{d}(\cdot) \theta_{d}\right]-\left(\dot{J} v+J \dot{v}_{r}\right) \\
=J M^{-1}\left[-\alpha J^{T} B \widetilde{e}_{B}-K_{v} r+Y_{d}(\cdot) \widehat{\theta}_{d}\right]
\end{gathered}
$$

or

$$
M \dot{r}+C(v) r+D(v) r+K_{v} r+\alpha J^{T}(\eta) B \widetilde{e}_{B}-Y_{d}(\cdot) \widetilde{\theta}_{d}=0,
$$

where $\widetilde{\theta}_{d}=\widehat{\theta}_{d}-\theta_{d}$ denotes the parameter estimation error. Next, the following nonnegative function is introduced to analyze the stability of the proposed control law:

$$
V=\frac{1}{2} r^{T} M r+\frac{1}{2} \widetilde{\theta}_{d}^{T} L_{d}^{-1} \widetilde{\theta}_{d}+\alpha P\left(\delta \eta_{B}\right)
$$

Differentiating $V$ with respect to time and using the update law (22) yield

$$
\dot{V}=r^{T} M \dot{r}-\widetilde{\theta}_{d}^{T} Y_{d}^{T}(\cdot) r+\alpha\left(\dot{B} \delta \eta_{B}+B \delta \dot{\eta}_{B}\right)^{T} \widetilde{e}_{B}
$$

Utilizing (17), (20), and (25) and cancelling the common terms lead to

$$
\dot{V}=-r^{T} D(v) r-r^{T} K_{v} r-\alpha^{2} \widetilde{e}_{B}^{T} \widetilde{e}_{B} \leq 0,
$$

where Property 3 is used. Now, a new theorem can be stated as follows.

Theorem 3. Given a closed-loop underwater vehicle with kinematically redundant $n$ thrusters in (25), the proposed adaptive control law (21) and parameter update law (22) guarantee the convergence of $\eta$ into a dynamic region $f\left(\delta \eta_{B}\right) \leq$ 0 in the sense that $\widetilde{e}_{B} \rightarrow 0$ and $r \rightarrow 0$, as $t \rightarrow \infty$. 
Proof. In view of the fact that $M$ is uniformly positive definite, $V$ in (26) is positive definite in $r, \widetilde{\theta}_{d}$. Since $V>0$ and $\dot{V} \leq 0, V$ is bounded. Hence, $r, \widetilde{\theta}_{d}$, and $f\left(\delta \eta_{B}\right)$ are bounded. The boundedness of $f\left(\delta \eta_{B}\right)$ ensures the boundedness of $\left(\partial f\left(\delta \eta_{B}\right) / \partial \eta_{B}\right)$. Hence, $\widetilde{e}_{B}$ in (11) and its derivative are bounded. The boundedness of $v$ guarantees the boundedness of $\dot{\eta}$ since $\dot{\eta}=J(\eta) v$ and $J(\eta)$ is a trigonometric function. The boundedness of $J(\eta)$ and $J^{-1}(\eta)$ leads to the boundedness of $u(\eta)$ and $u^{W \dagger}(\eta)$ in (19) and (20), respectively. Moreover, $\dot{v}_{r}$ in (16) remains a bounded signal by employing standard signal chasing arguments. From the closed-loop equation, it can be concluded that $\dot{r}$ in (17) is also bounded using the fact that the kinematic and dynamic terms denoted by $u(\eta), u^{W \dagger}(\eta)$, $C(v), D(v)$, and $g(\eta)$ are bounded for all possible $\eta(t)$ and $v(t)$. Thus, $\dot{v}$ is bounded since $\dot{r}=\dot{v}-\dot{v}_{r}$. Differentiating (28) with respect to time gives

$$
\ddot{V}=-r^{T} \dot{D}(v) r-2 \dot{r}^{T} D(v) r-2 \dot{r}^{T} K_{v} r-2 \alpha^{2} \dot{\tilde{e}}_{B}^{T} \widetilde{e}_{B} .
$$

Hence, $\ddot{V}$ is bounded since $\dot{r}, r, \dot{\tilde{e}}_{B}$, and $\widetilde{e}_{B}$ are all bounded. Therefore, $\dot{V}$ is uniformly continuous. Applying Barbalat's lemma [21] gives $\dot{V} \rightarrow 0$ which also indicates that $\widetilde{e}_{B} \rightarrow$ 0 and $r \rightarrow 0$. From (11), $\widetilde{e}_{B} \rightarrow 0$ indicates that $\widetilde{e}_{B}$ is approaching zero. Therefore, $\eta$ converges to the moving desired scalable region.

Remark 4. To keep the thruster force within the saturation limits, the diagonal elements of the weight matrix can be formulated as [10]

$$
W_{s}=\operatorname{diag}\left[h_{1}, h_{2}, \ldots, h_{n}\right],
$$

where $n$ is the number of thrusters and $h_{i}$ is given as follows:

$$
h_{i}=1+\left|\frac{\partial H}{\partial F_{i}}\right| \text {. }
$$

Note that the second term on the right hand side of (31) is obtained from differentiation of the thruster force variable $H$ with respect to the thrust force of the $i$ th thruster. The same concept was presented in [22], where a similar function based on joint position variables was used to avoid joint limits of a manipulator.

\section{Simulation Results}

In this section, two simulation studies are carried out to assess the effectiveness of the proposed fault-tolerant region-based control law for an underwater vehicle. The ODIN $[9,23]$ AUV is known as a near-spherical omnidirectional vehicle mounted with four horizontal thrusters and four vertical thrusters and was chosen for these numerical simulations. The estimated parameters in (22) are $80 \%$ to $90 \%$ of the original values in order to verify the adaptive performance of the proposed controller. Values for vehicle parameters such as hydrodynamic damping matrices, added masses, and the thruster configuration matrix are given in the appendix. Hydrodynamic damping is assumed to be the summation

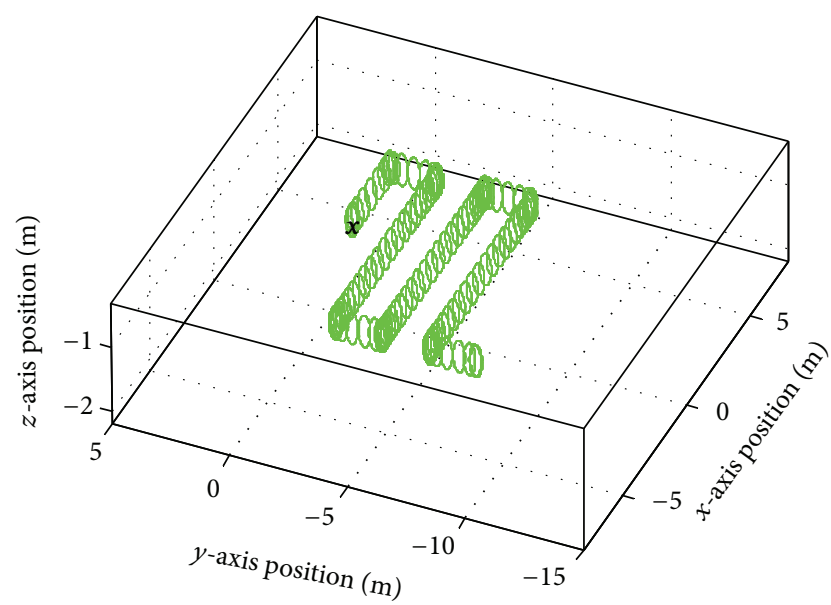

FIgURE 4: The desired trajectory for the region control scheme where " $x$ " marks the initial position of the AUV.

of linear and quadratic damping whose effects are noncoupled with respect to other velocities. The performance of conventional tracking control and the proposed technique is observed concerning two cases. The first case is with no thruster fault and the second case is where any one of the four horizontal thrusters is faulty and any one of the four vertical thrusters is faulty.

In these simulation studies, the following inequality function is defined:

$$
\begin{aligned}
f\left(\delta \eta_{B}\right)= & s_{x}\left(x-x_{0}\right)^{2}+s_{y}\left(y-y_{0}\right)^{2} \\
& +s_{z}\left(z-z_{0}\right)^{2}+s_{\phi}\left(\phi-\phi_{0}\right)^{2} \\
& +s_{\theta}\left(\theta-\theta_{0}\right)^{2}+s_{\psi}\left(\psi-\psi_{0}\right)^{2} \leq \kappa_{r}^{2},
\end{aligned}
$$

where the elements of $\left\{s_{x}, s_{y}, s_{z}, s_{\phi}, s_{\theta}, s_{\psi}\right\}$ are the components of the time-varying scaling matrix $B$ and $\kappa_{r}$ is a scalar tolerance. In these simulations, the matrix $B$ is defined as the identity matrix. Note that (32) can also be represented as the root mean square error for all axes in the following form:

$$
\begin{aligned}
& \left(s_{x}\left(x-x_{0}\right)^{2}+s_{y}\left(y-y_{0}\right)^{2}+s_{z}\left(z-z_{0}\right)^{2}+s_{\phi}\left(\phi-\phi_{0}\right)^{2}\right. \\
& \left.\quad+s_{\theta}\left(\theta-\theta_{0}\right)^{2}+s_{\psi}\left(\psi-\psi_{0}\right)^{2}\right)^{1 / 2} \leq \kappa_{r} .
\end{aligned}
$$

The ODIN vehicle is required to track a predefined trajectory as illustrated in Figure 4 where the green (cross-sectional lines) path is the horizontal basis position initialized at the position $\left[\begin{array}{lll}1.5 & 0 & -1.2\end{array}\right]^{T} \mathrm{~m}$. Moreover, the vehicle is initialized at the same position $\eta_{1}(0)=\left[\begin{array}{lll}1.5 & 0 & -1.2\end{array}\right]^{T} \mathrm{~m}$ while the initial values for attitude are set to be $\eta_{2}(0)=\left[\begin{array}{lll}0 & 0 & 0\end{array}\right]^{T}$ degrees. The attitude is kept constant for the entire motion. The control gains are set to the following:

$$
\begin{gathered}
k_{p}=28 ; \quad \alpha=1 ; \\
K_{v}=\operatorname{diag}\left(\left[\begin{array}{lllllll}
1.08 & 1.08 & 1.08 & 1.1 & 1.1 & 1.1
\end{array}\right]\right) .
\end{gathered}
$$




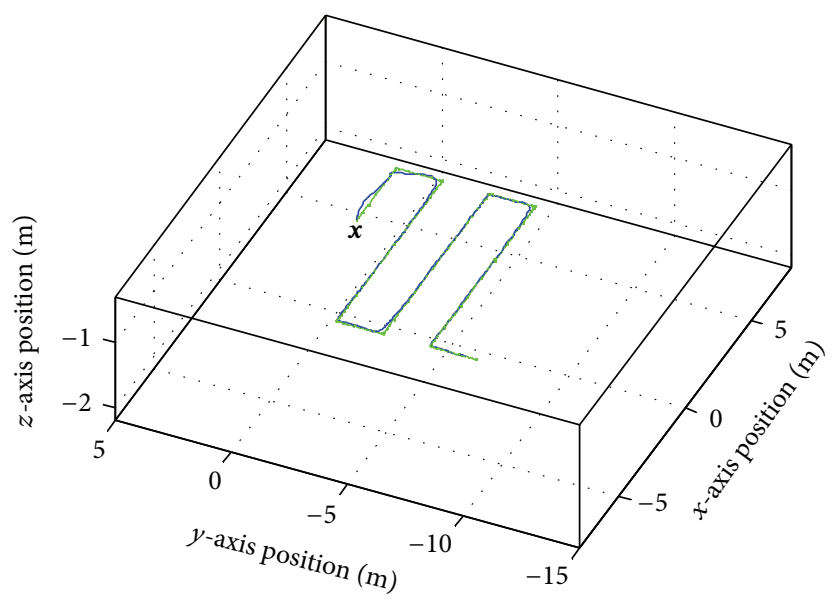

FIGURE 5: Three-dimensional view for conventional tracking control ( $\kappa_{r}$ is set to be arbitrarily small: $\kappa_{r}=0.001$ ) with no fault.

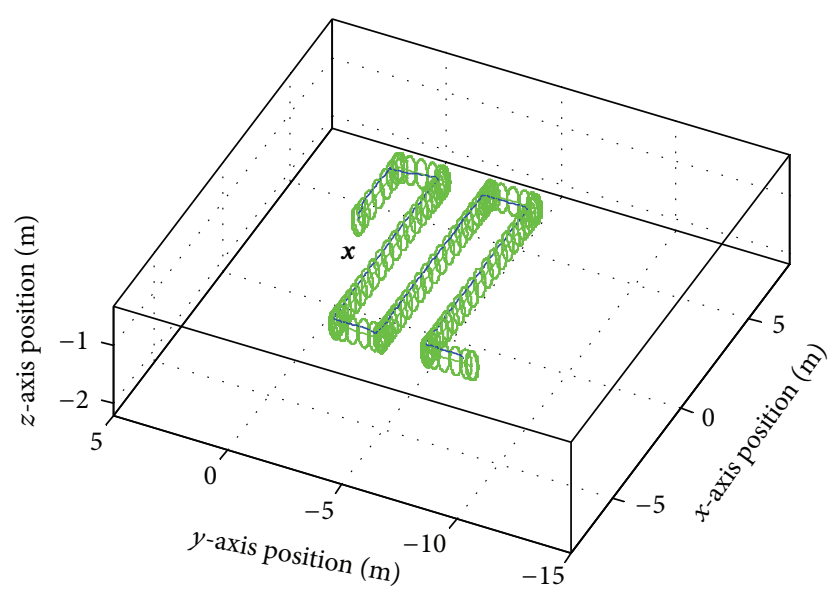

FIGURE 6: Three-dimensional view for region tracking control $\left(\kappa_{r}=\right.$ $0.25)$ with no fault.

The results from Case 1, where the weighted matrix is an identity matrix, are depicted in Figures 5 and 6. The vehicle represented by solid blue lines initially navigates to the desired line and desired region as can be seen in Figures 5 and 6 , respectively. The abrupt changes of errors at the beginning are caused by vehicle parameters' errors before it stays at the desired position even though the region is moving or scaling. The position of the AUV is indicated with solid blue lines. Meanwhile, the forces for all the thrusters of Case 1 using region tracking control law are given in Figure 7.

In Case 2, two thrusters on the AUV are faulty; one of the four horizontal thrusters and one of the four vertical thrusters stop functioning after $120 \mathrm{~s}$ of motion. The results are plotted in Figures 8 and 9. Even though the fault case happens during the motion, the thruster allocation forces are different from that of Case 1 and the position of the AUV is still kept inside the region. Figure 10 depicts the forces of functioning and faulty thrusters using region tracking control law at various time instants. The total required thruster forces are listed in Table 1, where all values are computed based on the Euclidean
TABLE 1: Simulation results for Case 2: required thruster forces.

\begin{tabular}{lcc}
\hline & $\begin{array}{c}\text { Conventional } \\
\text { tracking controller }\end{array}$ & $\begin{array}{c}\text { Proposed control } \\
\text { scheme }\end{array}$ \\
\hline Horizontal thrusters, (N) & 487.42 & 483.29 \\
Vertical thrusters, (N) & 229.53 & 218.43 \\
\hline Total input, (N) & 716.95 & 701.72 \\
\hline
\end{tabular}

norm for the entire time. The term "Total input" in the table is calculated by numerical summation of the norm of thruster forces and it can be interpreted as the total energy requirement. From Table 1, the region function formulation under the proposed control method needs significantly less energy than the conventional controller.

\section{Conclusion}

In this paper, a new adaptive fault-tolerant region-based tracking control scheme is proposed for an autonomous underwater vehicle with redundant thrusters. Two cases have been considered. The first case is with no thruster fault and the second case is where any one of the four horizontal thrusters is faulty and any one of the four vertical thrusters is faulty. Although some thrusters are faulty during task execution, the AUV is still able to track a desired moving region based on the thruster allocation forces. Moreover, it has been shown that the proposed technique can minimize the required thruster forces unlike the conventional adaptive tracking control. A Lyapunov-like function has been proposed for stability analysis. Simulation results have been presented to demonstrate the performance of the proposed controller.

\section{Appendix}

The omnidirectional intelligent navigator (ODIN) is a nearspherical AUV designed by the University of Hawaii. The dynamic model of ODIN is given by [9]

$$
\left[M_{\mathrm{RB}}+M_{\mathrm{A}}\right] \dot{v}+\left[C_{\mathrm{RB}}(v)+C_{A}(v)\right] v+D(v) v+g(\eta)=\tau,
$$

where the subscripts $\mathrm{RB}$ and $A$ represent the rigid body and added mass terms of the relevant parameters, respectively. The numerical values for the matrices of the vehicle dynamic equation (A.1) are given as

$$
M_{\mathrm{RB}}=\left[\begin{array}{cccccc}
m & 0 & 0 & 0 & m z_{G} & 0 \\
0 & m & 0 & -m z_{G} & 0 & 0 \\
0 & 0 & m & 0 & 0 & 0 \\
0 & -m z_{G} & 0 & I_{x x} & 0 & 0 \\
m z_{G} & 0 & 0 & 0 & I_{y y} & 0 \\
0 & 0 & 0 & 0 & 0 & I_{z z}
\end{array}\right]
$$

where $I_{x x}=I_{y y}=I_{z z}=I=(8 / 15) \pi \rho_{v} r_{\text {ODIN }}^{5}$ are the moments of inertia about the principle axes. 

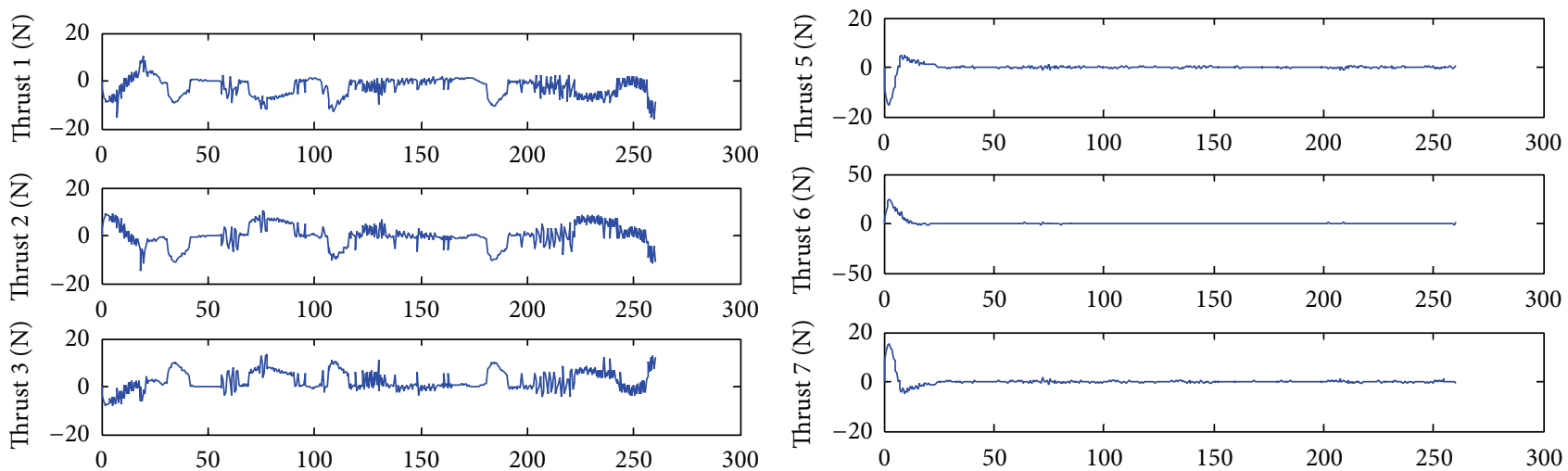

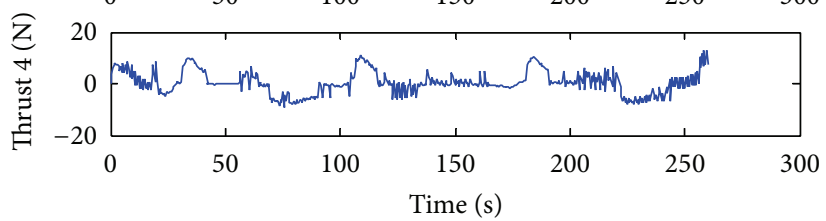

(a)

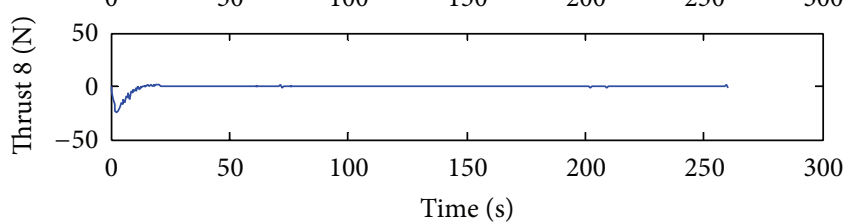

(b)

FIgURE 7: Thruster forces for Case 1 using proposed tracking control law. (a) Horizontal thrusters and (b) vertical thrusters.

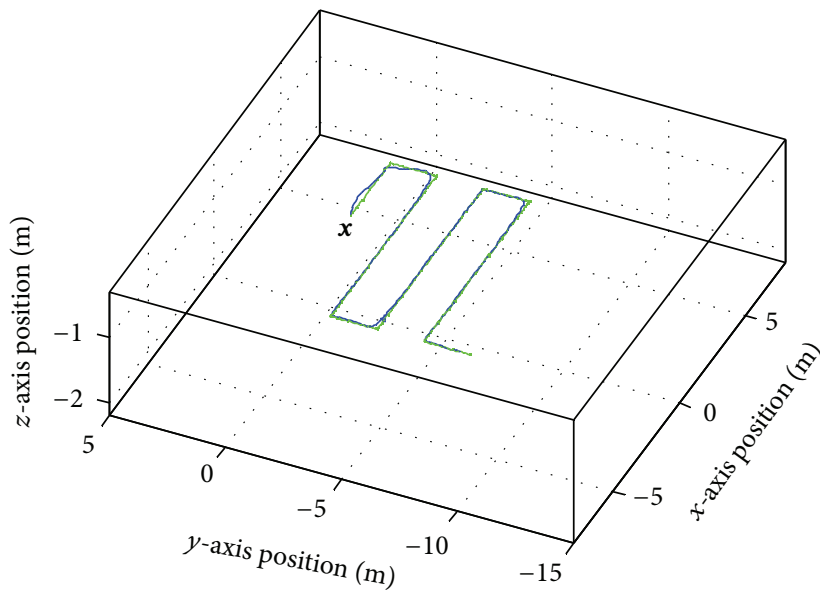

FIGURE 8: Three-dimensional view for conventional tracking control $\left(\kappa_{r}\right.$ is set to be arbitrarily small: $\left.\kappa_{r}=0.001\right)$ with fault.

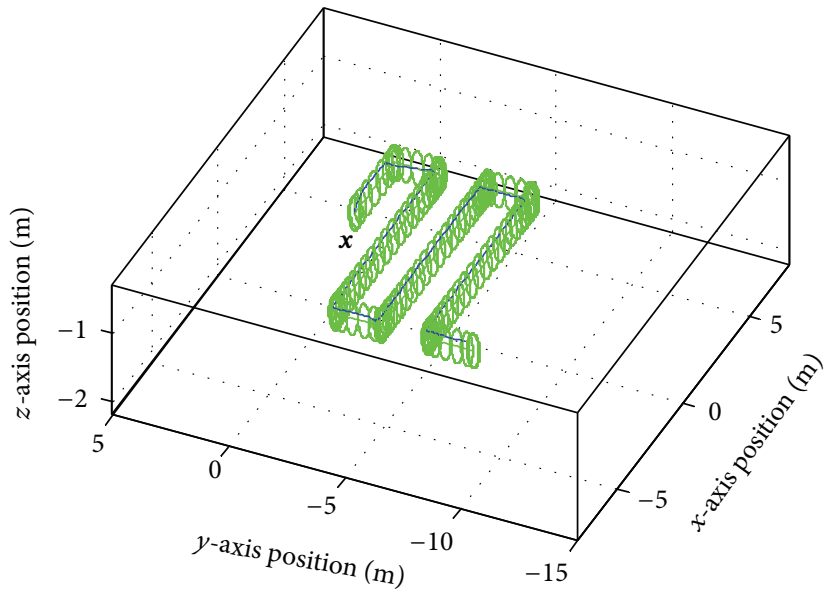

FIGURE 9: Three-dimensional view for region tracking control $\left(\kappa_{r}=0.25\right)$ with fault. 
Consider

$$
\begin{aligned}
& M_{A}=\left[\begin{array}{cccccc}
X_{u d} & 0 & 0 & 0 & 0 & 0 \\
0 & Y_{v d} & 0 & 0 & 0 & 0 \\
0 & 0 & Z_{w d} & 0 & 0 & 0 \\
0 & 0 & 0 & 0 & 0 & 0 \\
0 & 0 & 0 & 0 & 0 & 0 \\
0 & 0 & 0 & 0 & 0 & 0
\end{array}\right] \\
& C_{\mathrm{RB}}(v)=\left[\begin{array}{cccccc} 
& & & & -m u_{2} \\
0 & 0 & 0 & m z_{G} u_{6} & m u_{3} & m u_{1} \\
0 & 0 & 0 & -m u_{3} & m z_{G} u_{6} & 0 \\
0 & 0 & 0 & m\left(u_{2}-z_{G} u_{4}\right) & -m\left(u_{1}+z_{G} u_{5}\right) & -I u_{5} \\
-m z_{G} u_{6} & m u_{3} & -m\left(u_{2}-z_{G} u_{4}\right) & 0 & I u_{6} & I u_{4} \\
-m u_{3} & -m z_{G} u_{6} & m\left(u_{1}+z_{G} u_{5}\right) & -I u_{6} & 0 & 0 \\
m u_{2} & -m u_{1} & 0 & I u_{5} & -I u_{4} &
\end{array}\right] \text {, }
\end{aligned}
$$

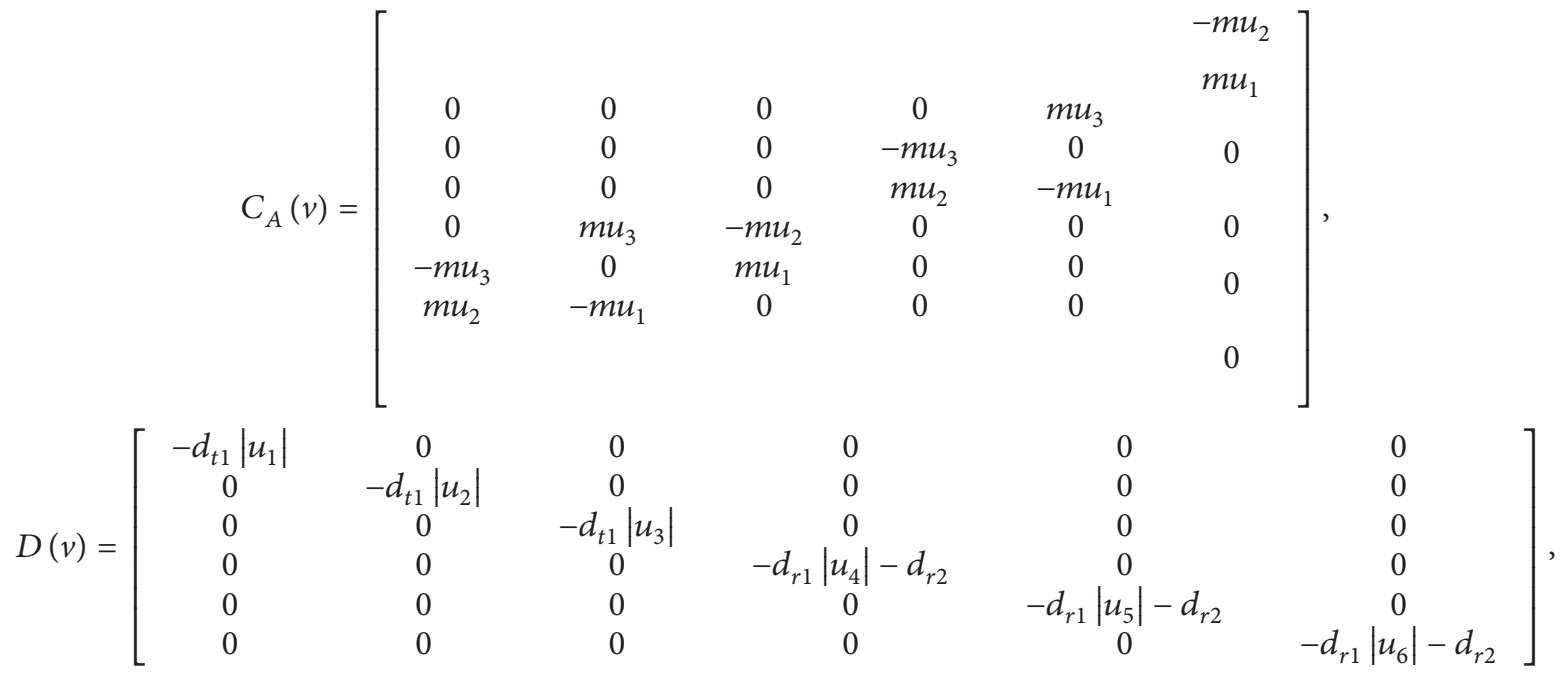

$$
\begin{aligned}
& g(\eta)=\left[\begin{array}{c}
\left(m \mathrm{~g}-\frac{4}{3} \pi r^{3} \rho \mathrm{g}\right) \sin (\theta) \\
-\left(m \mathrm{~g}-\frac{4}{3} \pi r^{3} \rho \mathrm{g}\right) \cos (\theta) \sin (\phi) \\
-\left(m \mathrm{~g}-\frac{4}{3} \pi r^{3} \rho \mathrm{g}\right) \cos (\theta) \cos (\phi) \\
z_{G} m g \cos (\theta) \sin (\phi) \\
z_{G} m \mathrm{~g} \sin (\theta) \\
0
\end{array}\right]
\end{aligned}
$$

Provided that $r_{\text {ODIN }}=0.31 \mathrm{~m}$ is the radius of ODIN, $m=125.0 \mathrm{~kg}$ is the mass of ODIN, $z_{G}=0.05 \mathrm{~m}$ is the distance of the center of gravity from the geometric center, $\rho_{v}=965 \mathrm{~kg} / \mathrm{m}^{3}$ is the average density of the ODIN AUV, and $\rho=1000 \mathrm{~kg} / \mathrm{m}^{3}$ is the density of fresh water, $\mathrm{g}=9.81 \mathrm{~m} / \mathrm{s}^{2}$. The hydrodynamic derivatives are given by $X_{u d}=Y_{v d}=Z_{w d}=(2 / 3) \pi \rho r_{\mathrm{ODIN}}^{3}$, the translational quadratic damping factor $d_{t 1}=-248 \mathrm{~N}(\mathrm{~s} / \mathrm{m})^{2}$, the angular quadratic damping factor $d_{r 1}=-280 \mathrm{Ns}^{2} / \mathrm{m}$, and the angular linear damping factor $d_{r 2}=-230 \mathrm{Ns}^{2} / \mathrm{m}$. The thruster configuration matrix is defined as follows: 

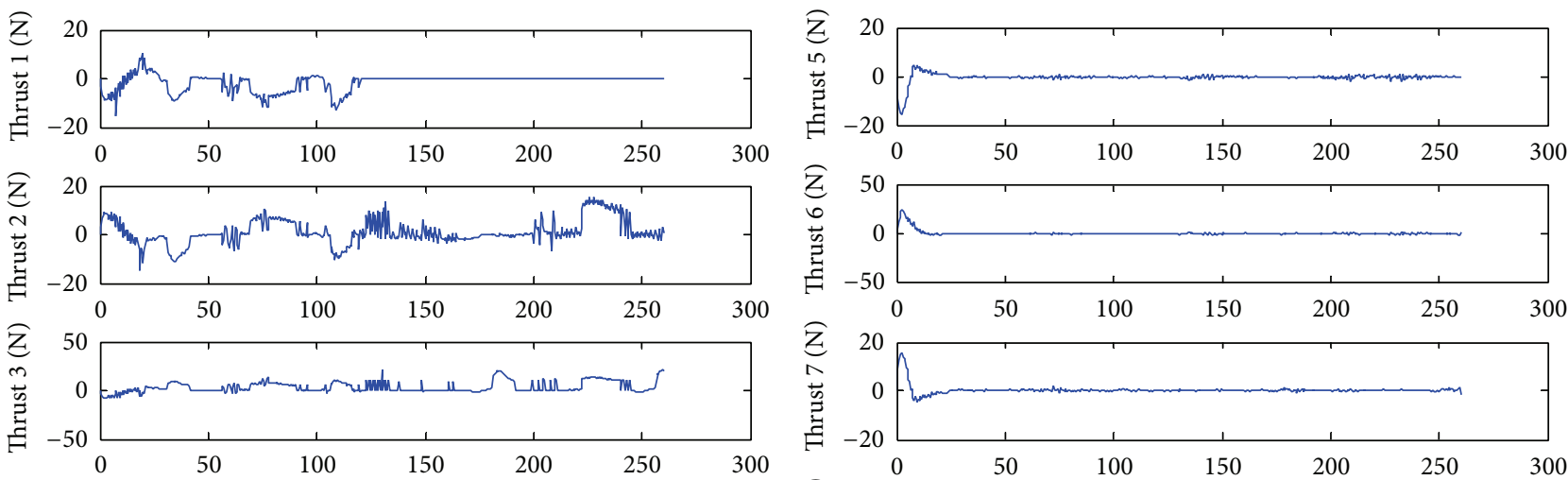

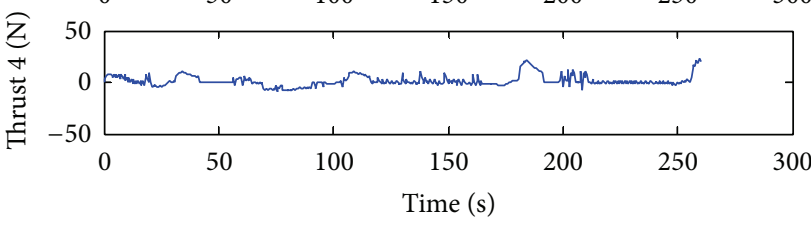

(a)

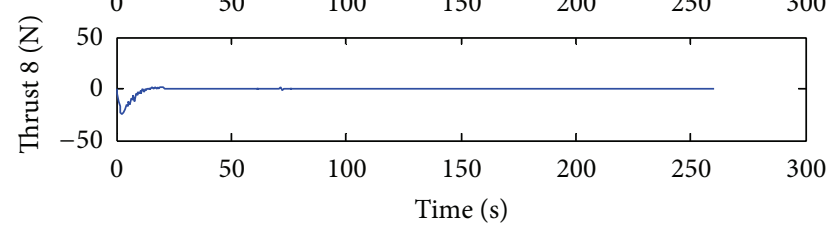

(b)

FIGURE 10: Thruster forces for Case 2 using proposed tracking control law. (a) Horizontal thrusters and (b) vertical thrusters.

$$
E=\left[\begin{array}{cccccccc}
s & -s & -s & s & 0 & 0 & 0 & 0 \\
s & s & -s & -s & 0 & 0 & 0 & 0 \\
0 & 0 & 0 & 0 & -1 & -1 & -1 & -1 \\
0 & 0 & 0 & 0 & R s & R s & -R s & -R s \\
0 & 0 & 0 & 0 & R s & -R s & -R s & R s \\
R_{z} & -R_{z} & R_{z} & -R_{z} & 0 & 0 & 0 & 0
\end{array}\right],
$$

where $s=\sin ((1 / 4) \pi), R=0.381 \mathrm{~m}$ denotes the distance from the center of the vehicle to the vertical thruster's center, and $R_{z}=0.508 \mathrm{~m}$ represents the radial distance from the center of the vehicle to the horizontal thruster's center.

\section{Conflict of Interests}

The authors declare that there is no conflict of interests regarding the publication of this paper.

\section{Acknowledgments}

The authors would like to thank Offshore Geo-Surveys (OGS) Sdn. Bhd. for their invaluable help and advice on the underwater vehicle. This work was supported in part by the Ministry of Higher Education, Malaysia, and Universiti Teknologi Malaysia under Grant no. R.J130000.7823.4F101 and Q.J130000.2509.05H54.

\section{References}

[1] J. Yuh, "Design and control of autonomous underwater robots: a survey," Autonomous Robots, vol. 8, no. 1, pp. 7-24, 2000.

[2] G. Antonelli, Underwater Robots: Motion and Force Control of Vehicle-Manipulator Systems, Springer, Germany, Berlin, 2003.
[3] O.-E. Fjellstad and T. I. Fossen, "Position and attitude tracking of AUV's: a quaternion feedback approach," IEEE Journal of Oceanic Engineering, vol. 19, no. 4, pp. 512-518, 1994.

[4] D. R. Yoerger and J.-J. E. Slotine, "Robust trajectory control of underwater vehicles," IEEE Journal of Oceanic Engineering, vol. 10, no. 4, pp. 462-470, 1985.

[5] K. P. Venugopal, R. Sudhakar, and A. S. Pandya, "On-line learning control of autonomous underwater vehicles using feedforward neural networks," IEEE Journal of Oceanic Engineering, vol. 17, no. 4, pp. 308-319, 1992.

[6] J. Yuh, "Learning control for underwater robotic vehicles," IEEE Control Systems Magazine, vol. 14, no. 2, pp. 39-46, 1994.

[7] F. Wang, Y. Xu, L. Wan, and Y. Li, "Modified learning of T$S$ fuzzy neural network control for autonomous underwater Vehicles," in Proceedings of the International Conference on Information Technology and Computer Science (ITCS '09), pp. 361-365, July 2009.

[8] X. Li, S. P. Hou, and C. C. Cheah, "Adaptive region tracking control for autonomous underwater vehicle," in Proceedings of the 11th International Conference on Control, Automation, Robotics and Vision (ICARCV '10), pp. 2129-2134, Singapore, December 2010.

[9] T. K. Podder and N. Sarkar, "Fault-tolerant control of an autonomous underwater vehicle under thruster redundancy," Robotics and Autonomous Systems, vol. 34, no. 1, pp. 39-52, 2001. 
[10] N. Sarkar, T. K. Podder, and G. Antonelli, "Fault-accommodating thruster force allocation of an AUV considering thruster redundancy and saturation," IEEE Transactions on Robotics and Automation, vol. 18, no. 2, pp. 223-233, 2002.

[11] D. Zhu, Q. Liu, and Y. Yang, "An active fault-tolerant control method ofunmanned underwater vehicles with continuous and uncertain faults," International Journal of Advanced Robotic Systems, vol. 5, no. 4, pp. 411-418, 2008.

[12] J.-K. Choi and H. Kondo, "On fault-tolerant control of a hovering AUV with four horizontal and two vertical thrusters," in Proceedings of the Conference on Oceans (OCEANS '10), Sydney, Australia, May 2010.

[13] Q. Liu, D. Zhu, and S. X. Yang, "Unmanned underwater vehicles fault identification and fault-tolerant control method based on FCA-CMAC neural networks, applied on an actuated vehicle," Journal of Intelligent and Robotic Systems: Theory and Applications, vol. 66, no. 4, pp. 463-475, 2012.

[14] M. Motamedi, M. T. Ahmadian, G. Vossoughi, S. M. Rezaei, and M. Zareinejad, "Adaptive sliding mode control of a piezoactuated bilateral teleoperated micromanipulation system," Precision Engineering, vol. 35, no. 2, pp. 309-317, 2011.

[15] M. Zeinali and L. Notash, "Adaptive sliding mode control with uncertainty estimator for robot manipulators," Mechanism and Machine Theory, vol. 45, no. 1, pp. 80-90, 2010.

[16] M. Roopaei, B. R. Sahraei, and T.-C. Lin, "Adaptive sliding mode control in a novel class of chaotic systems," Communications in Nonlinear Science and Numerical Simulation, vol. 15, no. 12, pp. 4158-4170, 2010.

[17] The Society of Naval Architects and Marine Engineers (SNAME), "Nomenclature for treating the motion of a submerged body through a fluid," in Technical and Research Bulletin, pp. 1-15, 1950.

[18] T. I. Fossen, Guidance and Control of Ocean Vehicles, John Wiley and Sons, New York, NY, USA, 1st edition, 1994.

[19] Z. H. Ismail and M. W. Dunnigan, "Tracking control scheme for an underwater vehicle-manipulator system with single and multiple sub-regions and sub-task objectives," IET Control Theory and Applications, vol. 5, no. 5, pp. 721-735, 2011.

[20] S. Soylu, B. J. Buckham, and R. P. Podhorodeski, "A chatteringfree sliding-mode controller for underwater vehicles with faulttolerant infinity-norm thrust allocation," Ocean Engineering, vol. 35, no. 16, pp. 1647-1659, 2008.

[21] J. J. E. Slotine and W. Li, Applied Nonlinear Control, Prentice Hall, 1991.

[22] T. F. Chan and R. V. Dubey, "Weighted least-norm solution based scheme for avoiding joint limits for redundant joint manipulators," IEEE Transactions on Robotics and Automation, vol. 11, no. 2, pp. 286-292, 1995.

[23] S. K. Choi, J. Yuh, and N. Keevil, "design of omni-directional underwater robotic vehicle," in Proceedings of the Conference on Oceans (OCEANS '10), pp. I192-I197, October 1993. 


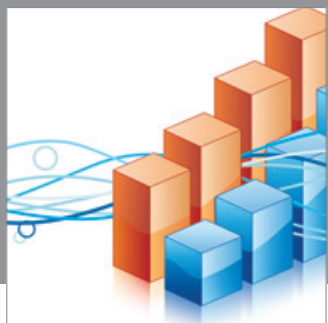

Advances in

Operations Research

mansans

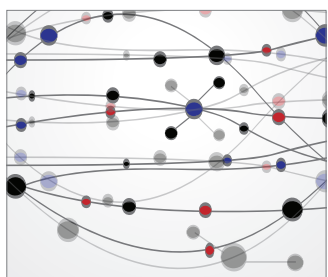

The Scientific World Journal
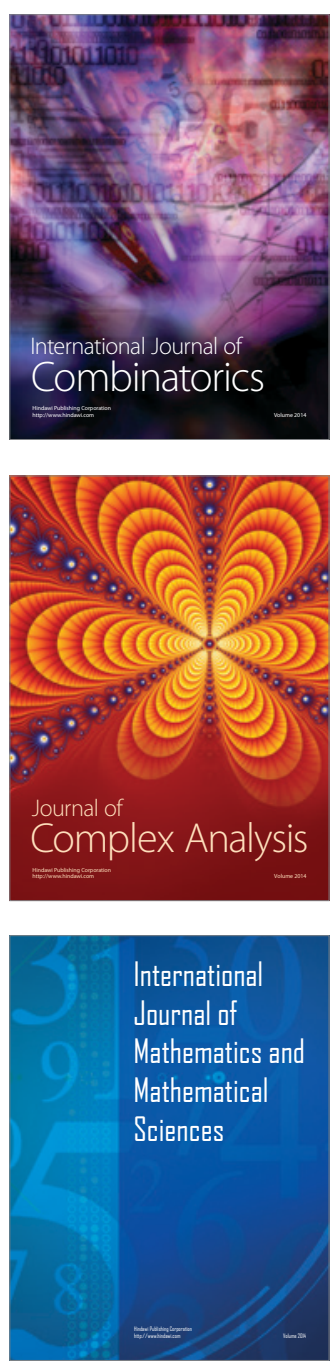
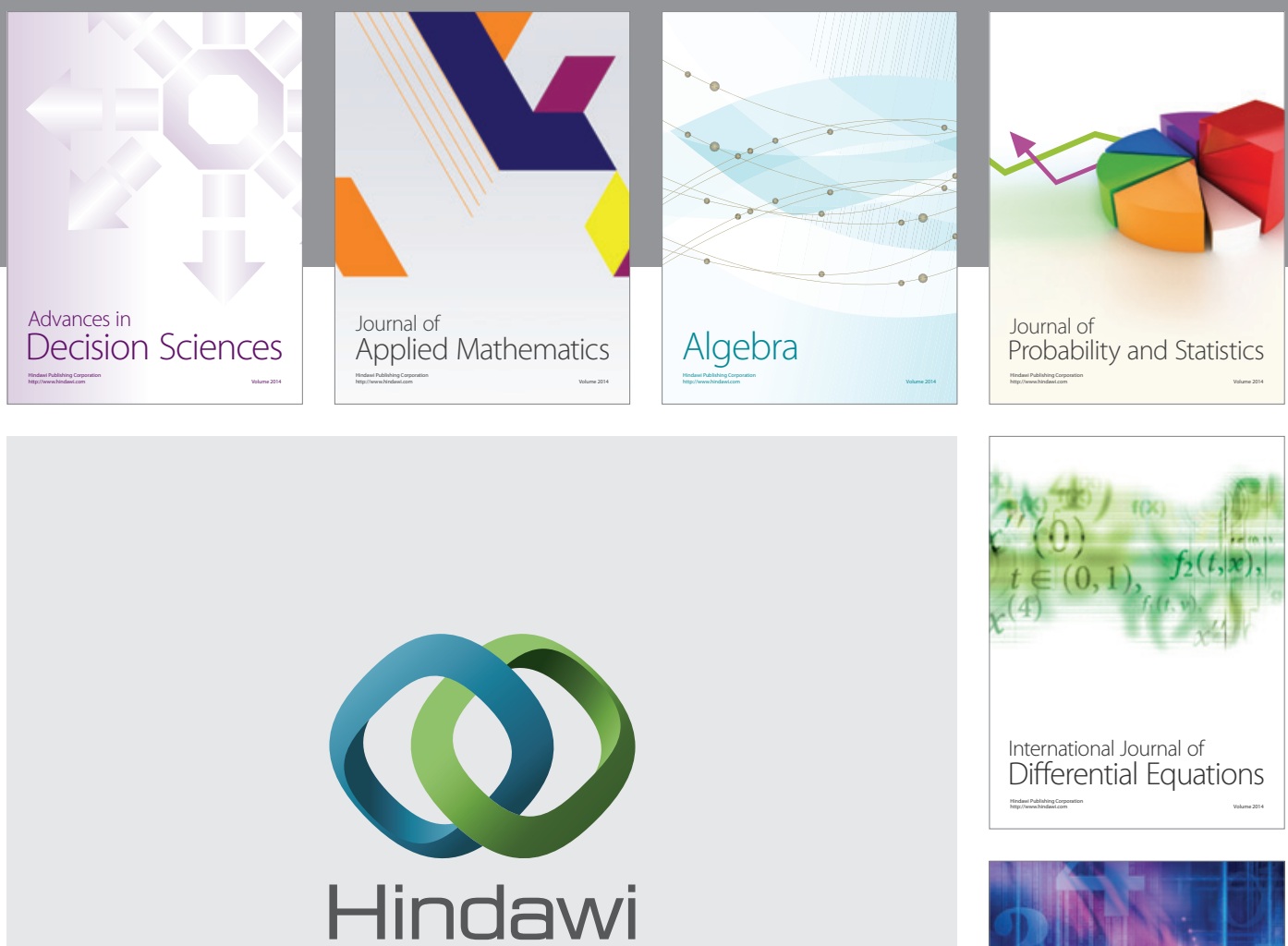

Submit your manuscripts at http://www.hindawi.com
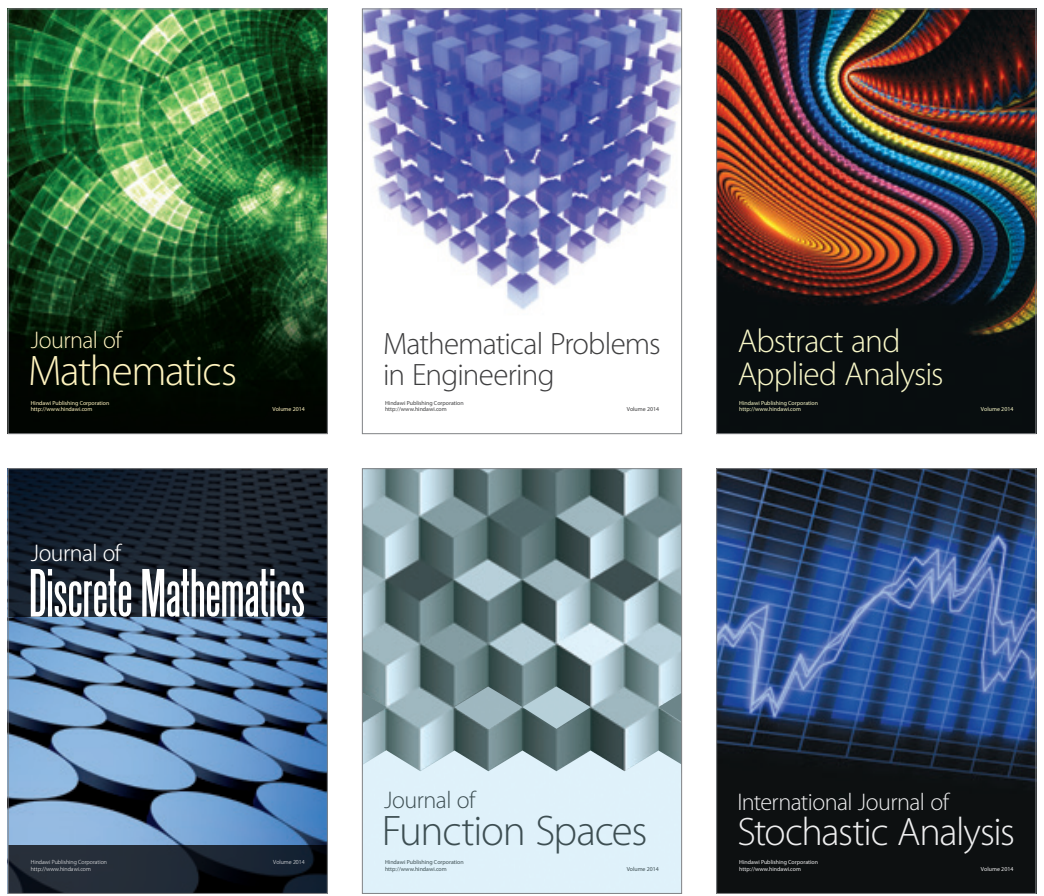

Journal of

Function Spaces

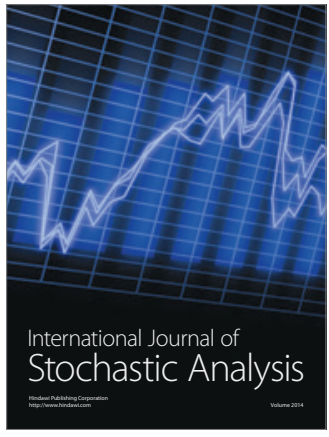

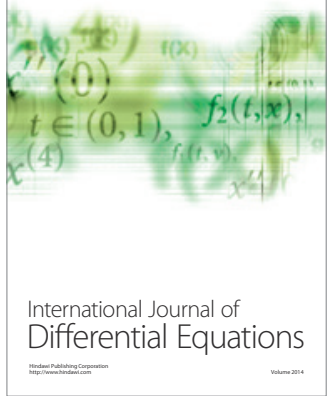
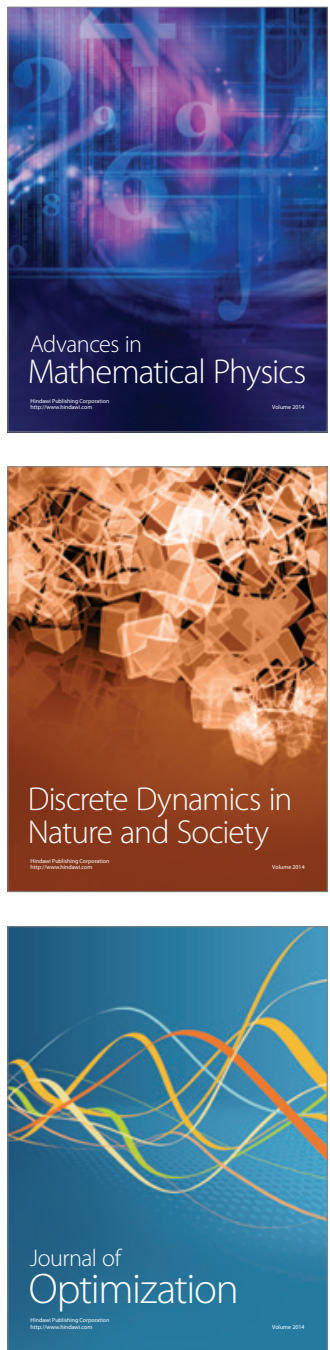